\title{
Analyse des Berner Codex 350, ein bibliographischer Beitrag zur chinesischen Medizin und zu deren Kenntnis bei Fabricius Hildanus und Haller
}

\author{
Von Erich Hintzsche, Bern
}

Im Zusammenhang mit Berichten über medizinische Literatur aus China erwähnt Albrecht von Haller (1774) in seiner Bibliotheca anatomica (Vol.I) mehrfach den Namen des ehemaligen Berner Stadt-Wundarztes GuiL. Fabricius Hildanus. Das umfassende Interesse dieses bedeutendsten deutschen Chirurgen des ausgehenden 16. und beginnenden 17. Jahrhunderts ist zwar in Fachkreisen bekannt, speziell soweit es naturwissenschaftliche und allgemein-ärztliche Fragen betrifft. Völlig neu aber ist, daß FABricıus sich auch mit dem Bildmaterial aus chinesischen medizinischen Büchern beschäftigt hat. Hallers darauf bezügliche Hinweise müssen unsere Aufmerksamkeit um so mehr erwecken, als die ersten in Europa gedruckten Werke über dieses Wissensgebiet nicht vor dem letzten Viertel des 17. Jahrhunderts publiziert wurden, also erst mehrere Dezennien nach dem Tode von Fabricius. Woher konnte dieser chinesische Literatur erhalten und wie konnte er sie sich zugänglich gemacht haben?

Haller nennt als Quelle seiner Hinweise die unveröffentlichten Briefe von Fabricius Hildanus ${ }^{1}$. Er lernte diese während seiner Tätigkeit als Bibliothekar in Bern (Mai 1735 bis 1736) kennen, zitierte aber später anscheinend aus der Erinnerung, denn weder seine handschriftlichen Auszüge ${ }^{2}$ noch deren anonym erschienene Veröffentlichung ${ }^{3}$ enthalten entsprechende Notizen. Im ersten Bande der Bibliotheca anatomica schrieb HAller auf S.9 nach anderen Berichten über anatomische Kenntnisse der Chinesen, daß in einem FABricius zugesandten Buche noch unbeachtet gebliebene anatomische Abbildungen vorhanden seien. Auf S.138 desselben Bandes wird diese Angabe in ähnlicher Form wiederholt, denn dort steht: Auch Fabricius Hildanus ist ein chinesisch geschriebenes Buch mit anatomischen Abbildungen übersandt worden.

\footnotetext{
1 Burgerbibliothek Bern, Cod. 495-497.

2 Burgerbibliothek Bern, Mss. Haller 21.

${ }^{3}$ Relationes de libris novis Fasc. XII p. 429-454. Gottingae 1754.
} 
Einer Abklärung der Herkunft dieses chinesischen Werkes glaubte ich schon nahe zu sein, als ich im nachgelassenen Briefwechsel von FABRICIUS in einem Schreiben an G. Horst in Ulm vom 26. August $1630^{4}$ die Stelle fand: «Mein Sohn Johannes, den Du im Jahre 1612 in Gießen ${ }^{5}$ bei mir gesehen hast, ist gottlob vor wenigen Monaten aus Indien nach Holland zurückgekehrt. Will's Gott, so wird er in Kürze hierher (d.h. nach Bern) kommen.» Johannes FABRY ist also als Wundarzt, möglicherweise im Dienste der Holländischen oder der Englischen Ostindischen Kompanie, bis nach Indien oder vielleicht sogar noch weiter in den Fernen Osten gekommen. War es nicht naheliegend, anzunehmen, daß er seinem Vater ein fremdes Werk medizinischen Inhaltes von dort gesandt oder mitgebracht hätte?

Blättert man aber weiter in der Briefsammlung des FABRICIUS, so stößt man bald auf die richtige Spur. Das von HaLler erwähnte chinesische Buch gehörte gar nicht FABRIcIUS, sondern stammte aus der Bibliotheca Bongarsiana. FABRICIUS schrieb nämlich seinem ehemaligen Schüler und Hausgenossen Dr.Johannes Burgauer nach Schaffhausen am 1.September $1632^{6}$ u.a.: "Übrigens habe ich in diesen Tagen aus einem in China gedruckten Buch der Bibliothek des kenntnisreichen, edlen und gelehrten Mannes Jacob Bongars de Bauldry, des ehemaligen Gesandten ${ }^{7}$ Heinrichs des Großen, erfahren, daß die Medizin bei den Chinesen auch in hohem Ansehen steht, denn es enthält viele, teils anatomische, teils botanische Figuren, aber sie sind so wenig fein und so klein geschnitten, daß ich nicht eine einzige Pflanze bestimmt habe erkennen können. Beschreibungen und Erklärungen sind beigegeben, aber in ihrer Sprache, die hier keiner der gelehrten Männer weder lesen noch verstehen kann. An den anatomischen Figuren aber habe ich bemerkt, daß die Fingernägel gleich den Krallen der Vögel spitz endigen. An den Füßen dagegen sind sie abgeschnitten, wie

${ }^{4}$ Burgerbibliothek Bern, Cod. 495/13. Hier und im folgenden sind alle lateinisch geschriebenen Stellen möglichst wortgetreu übersetzt.

${ }^{5}$ Auf der Rückreise von Hilden, wo FABRIciUs seiner Mutter während ihrer letzten Krankheit Hilfe zu bringen gesucht hatte (G. BECKER, 1957).

${ }^{6}$ Burgerbibliothek Bern, Cod. 495/59. Die Jahreszahl fehlt im Original, wie schon Haller in der Publikation (1754, S. 438) bemerkte. In dem Brief Nr. 59 ist aber auf ein vorhergehendes Schreiben vom 8. April Bezug genommen, das durch einen Herrn Stouchard überbracht worden sei. Dieser Brief ist in Abschrift unter Nr. 65 vorhanden und vom 8. April 1632 datiert, woraus sich die Richtigkeit der genannten Jahreszahl ergibt.

7 JACов Bongars (1554 bis 1612) nannte sich nur Geschäftsträger des Königs Heinrich IV. von Frankreich. Sein Vater war Seigneur de Bauldry et de la Chesnaye bei Orléans. Die Geschichte der Bibliotheca Bongarsiana ist beschrieben von H. BLöscH (1932). 
Du an den beigefügten aus dem erwähnten Buche genau abgezeichneten Figuren sehen kannst. Daraus wird deutlich, daß der Zeichner oder der Holzschneider nicht geirrt haben, sondern daß diese Gewohnheit bei jenem Volke besteht. Dieses Buch aber halte ich für sehr alt. Heute nämlich sind, weil die Holländer jenes. Volk seit vielen Jahren besuchten, die Drucke bei diesem mehr verfeinert, wie mir ehedem in Holland berichtet wurde.»

Bald danach schickte FABRICIUS auch die versprochenen Zeichnungen ${ }^{8}$. Er schrieb dazu: «Ich möchte nämlich, daß Du die eine oder andere anatomische Figur aus dem chinesischen Buche siehst und Dein Urteil über diese - wegen der Unterschiede der Finger- und Zehennägel - schreibst, was ich Dich bitte, in Muße zu tun.»

Dr. Burgauer nahm sich Zeit für seine Antwort, sie datiert vom 31. Juli $1633^{9}$. In dem sehr langen, zum Teil die Sätze von Fabricius wörtlich wiederholenden Schreiben kommt er zu dem Schluß, sich bei dem Fehlen genauerer Angaben lieber eines Urteils enthalten zu wollen. Er verweist auf die Beschreibung der Nägel bei GALEN, erwähnt die geringe Eignung so langer und zugespitzter Fingernägel für handwerkliche Arbeiten, z.B. für die Anfertigung feiner Porzellanwaren und führt an, daß kein Schriftsteller unter den körperlichen Eigenschaften der Chinesen spezielle Befunde an den Nägeln hervorgehoben habe. Als einzige Quelle seiner sonstigen Kenntnisse ist Mercator genannt, bei dem schon zu lesen wäre, die Kunst des Druckens sei in China so alt, daß man sich des Erfinders nicht mehr erinnern könne.

Fabricius scheint sich mit dieser ausweichenden Auskunft begnügt zu haben; seine weiteren Briefe aus der damaligen Zeit lassen erkennen, daß er kränkelte und die Mühen des Alters verspürte. Er hätte vielleicht gern eine Notiz über die Beobachtungen an den chinesischen Zeichnungen in die 2.Auflage seines Buches Vom Nutzen der Anatomie eingefügt, mit deren Vorbereitung er bis an sein Lebensende beschäftigt war ${ }^{10}$; es finden sich aber keine Briefstellen oder andere Hinweise auf derartige Bemühungen mehr.

${ }^{8}$ Burgerbibliothek Bern, Cod. 495/62, undatiert. Die verspätete Zusendung war durch die Abwesenheit des Zeichners bedingt. Die Abbildungen selbst (Hand und Unterschenkel mit Fuß aus dem chinesischen Buch) finden sich in Cod. 4.97 fast am Schluß des Bandes. Sie sind von der Rückseite durchgepauste Umrißzeichnungen, daher seitenverkehrt, aber in Form und Größe absolut der Vorlage gleich.

${ }^{9}$ Burgerbibliothek Bern, Cod. 495/66 und 67. Haller (1754, S. 438) datiert den Brief auf August 1633, übersah aber wohl das Wort "pridie» vor Kal.Sextileis.

10 Posthum publiziert in Veröffentlichungen der Schweizerischen Gesellschaft für Geschichte der Medizin und der Naturwissenschaften, Band 10 (1936). 
Bedeutsam bleibt indessen, festzustellen, welches chinesische Werk FABRICIUS in Händen hatte, denn es muß sich um eines der ersten nach Europa gekommenen medizinischen Bücher aus China gehandelt haben. Jedenfalls war es im Jahre 1612 - beim Tode von Bongars - bereits in dessen Besitz. Man wird sich auch fragen, welches Interesse ein Diplomat an einem solchen Werk haben konnte. Dazu ist zu bemerken, daß Bongars als $\mathrm{Hu}$ manist und Bücherliebhaber keineswegs einseitig war. Sprachliche und historische Werke machten zwar einen großen Teil seiner Bibliothek aus, doch waren auch die übrigen Fakultäten gut vertreten. Die Medizin stand z.B. mit 179 Büchern klassischer Schriftsteller gegenüber den 193 juristischen Bänden kaum zurück. Was weiter auffällt, ist ein unverkennbares Interesse für den Fernen Osten. In der Sammlung der Geschichtswerke findet sich unter den Jesuitica eine Reihe bemerkenswerter Missionsberichte aus Japan, China und Indien, ferner enthält ein Codex seiner Sammlung zusammen mit anderen Orientalia den ins Französische übersetzten Reisebericht von Marco Polo. Das in chinesischen Charakteren gedruckte Buch aus der Bibliothek Bongars steht also nicht ganz so vereinzelt da, wie es zunächst den Anschein hat.

Mehr als dreihundert Jahre mußten aber vergehen, ehe Titel und Inhalt näher untersucht wurden, und das, trotzdem es an wiederholten Hinweisen auf den als Codex 350 bezeichneten Band nicht gefehlt hat. Im ersten handschriftlichen Katalog der Berner Büchersammlung von Hontivus (1634) wird er als Liber anatomicus et botanicus ex China angeführt. Sinner (1760 bis 1772) hat den Band in seinem Verzeichnis der Berner Bibliothek nicht genannt, dagegen suchte ihn HALLER im 18. Jahrhundert durch die obenerwähnten Zitate bekanntzumachen. Im 19.Jahrhundert war es HAGEN (1875), der als Verfasser des Kataloges der Berner Handschriften auf das chinesische Buch hinwies. Trotzdem es sich um ein Druckwerk handelt, war es, wie erwähnt, von altersher bei den Codices eingereiht und fand so seinen Platz in dem eben genannten Verzeichnis. Auch Hagen kam jedoch nicht über die Bezeichnung «Liber sinesicus» hinaus. Schließlich wäre noch ein Zitat aus unserem Jahrhundert zu erwähnen; H. Strahm (in Blösch, 1932) schrieb in einem Bericht über besondere Werke aus der Bibliothek von JACOB Bongars : «Eine Merkwürdigkeit ist ein anatomisches und botanisches Buch aus China.» Alle diese Hinweise genügten jedoch nicht, um das Werk in Fachkreisen bekanntwerden zu lassen. Erst nachdem es mir 1957 gelungen war, einige noch unvollständige Angaben über den anatomischen Teil des Buches zu publizieren, fand es Interesse; es wurde deshalb als eines 
der ersten chinesischen Bücher medizinischen Inhaltes, die nach Europa kamen, in die Ausstellung «Médecine en Extrème Orient» (im Rahmen der Entretiens de Bichat, Paris September-Oktober 1959) aufgenommen.

Ein altchinesisches Werk nicht mehr im Originaleinband vor sich zu sehen, erweckt bei jedem Bücherliebhaber gewiß Bedauern. Offenbar aus dem Bestreben, dem Bande größere Haltbarkeit zu sichern, wurde der Codex 350 nämlich gleich nach der Übergabe an die Berner Bibliothek neu in Pergament eingebunden. Belege dafür sind das Vorsatzblatt mit dem Berner Wappen als Wasserzeichen und mehrere der auf dem Buchdeckel angebrachten Goldprägungen, die sich auf anderen Einbänden derselben Zeit wiederholen. Im neuen Buchblock wurden aber damals die ursprünglich in der Mitte gefalteten Blätter ausgebreitet und nur an einer Schmalseite geheftet, außerdem sind Teile von zwei ganz verschiedenen Werken in einem Band vereinigt. Dabei passierte dem Buchbinder das Mißgeschick, daß er die Blätter des einen Teiles richtig, die des anderen aber der üblichen Folge entgegengesetzt ordnete ${ }^{11}$. So befinden sich die Anfänge beider Werke jetzt in der Mitte des Bandes, was die Untersuchung zunächst etwas erschwerte.

Über den ersten die anatomischen Bilder enthaltenden Teil kann ich meine 1957 gemachten Angaben nun erweitern und gleichzeitig auch verbessern. Es handelt sich um das erste Buch (Chüan) einer neuen Auflage des Werkes Wan Ping Hui Ch'un, d.h. «Heilung aller Krankheiten » von Kuvg T'Ing-hSien. Die früher nach Wong und Wu (1932, S.137) angegebene Übersetzung des Titels «All diseases return to spring» habe ich neuerdings in noch engerer Anlehnung an die chinesische Formulierung übertragen gesehen als «Der nach 10000 Krankheiten wiederkehrende Frühling》 (W ALLNöfER und v. RotTAuscher, 1959, S.173). Wan = 10000 hat aber als höchstes der alten Zahlzeichen zugleich auch die Bedeutung «viele, alle» und die beiden Silben Hui Ch'un sind zum Begriffe «Heilung» zusammenzuziehen (Mathews, 2309/48), In diesem Sinne finde ich den Titel bei Huard und Wong (1956, S.188, und 1959, S.58) übersetzt «De la guérison des maladies». Das Titelblatt wiederholt in den fünf unteren Zeichen der letzten Spalte den Titel sogar unter Einfügung der Silbe Chin, was «Völlige Heilung aller Krankheiten» heißen würde.

Das Jahr der 1. Auflage des Wan Ping Hui Ch'un ist mir nicht genau bekannt, Wong und Wu (1936, S.238) geben dafür 1581 an. Nach Huard

${ }^{11}$ Dieses Versehen ist um so auffälliger, als in beiden Teilen von alter Hand jedes 5. Blatt - mit wenigen Mißzählungen - teils in römischen, teils in arabischen Ziffern numeriert ist. 
und Wong (1956, S.188) ist das Buch im 15.Jahr der Regierungsperiode Wan-li zusammengestellt und 1587 wieder herausgegeben. Wenn diese Angabe richtig ist, müßte es aber bereits im Jahre des Erscheinens wieder neu aufgelegt worden sein, denn das 15. Jahr der Periode Wan-li ist 1587. Sicher ist, daß das Berner Exemplar aus dem Wintermonat des 33. Jahres der Periode Wan-li, d.h. aus dem Jahre 1605, stammt. Diese Angabe ist völlig klar in den oberen 6 Zeichen der mittleren Spalte des Titelblattes enthalten, sie lauten Wan-li Ping-shên Tung-yüeh. Endlich ist der 7.Zeile von fol. $1 \mathrm{r}$ des Textes (Abb.1) zu entnehmen, daß das Buch in Nanking im Buchladen Tui Feng Chou verlegt war.

Die Druckstöcke sind 283 bis $290 \mathrm{~mm}$ breit und 202 bis $209 \mathrm{~mm}$ hoch. Auf den ersten acht Blättern, dem Vorwort, stehen außer der Faltzeile zweimal 6 Zeilen zu 14. Charakteren, ein weiteres Vorwort (fol. 9 bis 12) hat zweimal 7 Zeilen zu 14 Charakteren. Es folgen 4 fol. «Hinweise für den Leser » (Fan li) mit Faltzeile und zweimal 8 Zeilen zu 16 Charakteren, dann ein schlecht gedrucktes Register zu Buch 1 bis 8 mit Faltzeile und zweimal 13 Zeilen zu 24. Charakteren, bis hier alles ohne Interpunktion. Der am Ende offensichtlich unvollständige Text des ersten Buches umfaßt fol. 1 bis 48 , jedes außer der Faltzeile gleichfalls zu zweimal 13 Zeilen mit 24 Charakteren mit Interpunktion. An Abbildungen sind beigegeben: Fol. 34v der menschliche Körper von vorn, fol. 35 menschlicher Körper von hinten und Rumpf mit Darstellung der Eingeweide von der Seite, fol. 36 Hand und Unterschenkel mit Fuß.

Etwas verwirrlich werden die sonst bei verschiedenen Autoren gut übereinstimmenden Angaben über den Verfasser durch die Zitate im Katalog von M. Courant (1910). Er führt unter den Nummern 5102-03 (2.Band, S.85) in französischer Transkription an: «Sin khan oan ping hoei tchhoen. Traité des maladies. Par Kong Yun-LIN; complété et publié par son fils Kong Tseu-tshai. » Von dieser neuen Auflage besitzt die National-Bibliothek Paris das zweite, dritte und fünfte Buch, letzteres am Ende unvollständig, in einem Einband aus dem 18. Jahrhundert. Daß es sich dabei um ziemlich alten Bestand handelt, ergibt der Hinweis auf Fourmont (1742), der das Werk unter der Nummer CCCXIX mit dem Titel «Ván pím» anführte. Eine Datierung fehlt sowohl bei Courant wie bei Fourmont, was sich aus dem Mangel eines Titelblattes leicht erklärt. Im übrigen nennt Courant weitere Werke desselben Verfassers unter den Nummern 5101 und 5104-08. In französischer Transkription heißt der Autor danach: Kong Yun-Lin, Kong Sin von Kin khi oder zusammengezogen Kong Sin 


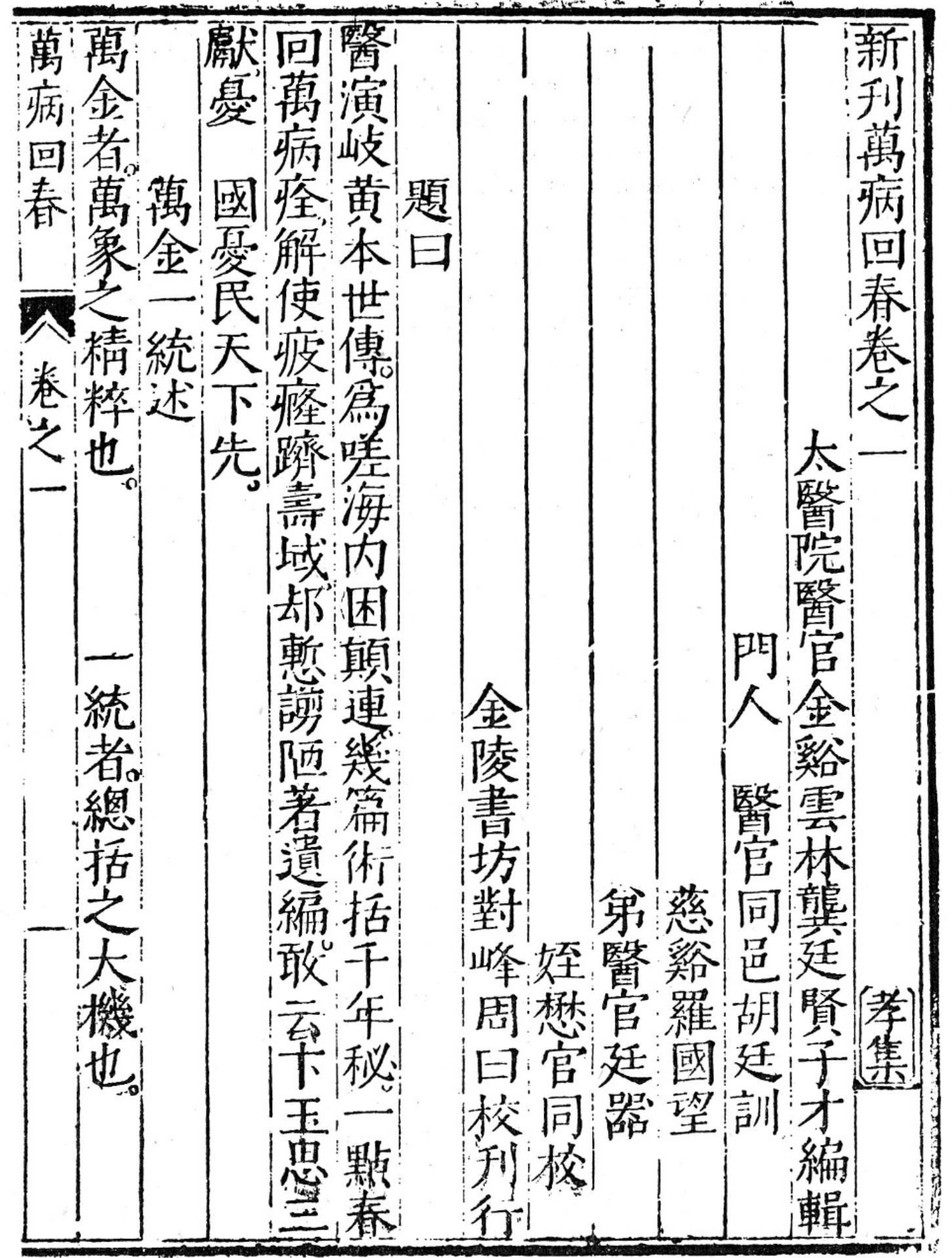

Abb. 1. Fol. 1r des ersten Buches des Wan Ping Hui Ch'un von Kung T'Ing-HSIEN nach der Auflage von 1605 
Yun-LIN; sein Sohn ist Kong Thing-hien mit dem Übernamen Tseu-tshai. Vorreden zu den einzelnen Werken sind von 1589 und 1591 datiert. Dem Berner Druck gehen Vorreden aus den Jahren 1597, 1596 und 1587 voraus. Die Benennung des Buches und der Autorname entsprechen auf der ersten Textseite (Abb.1) besser den Angaben des Pariser Kataloges von Courant als das eigentliche Titelblatt. Die 1. und 2.Zeile der Abb.1 heißen (in englischer Transkription): Hsin kan Wan Ping Hui Ch'un chih $i$ (Titel mit Hinweis auf neue Ausgabe und Buch 1); T'ai I-yuan I-kuan Chin ch'i Yunlin Kung T'ing-hsien Tzu-tsai Pien chi (Stellung, Herkunft, Name und Übername des Verfassers und Herausgebers der Kompilation). In den nächsten Zeilen folgen die Bearbeiter der neuen Auflage. Wie ich durch Vergleich in der Bibliothèque nationale feststellen konnte, gehören das in Bern liegende erste Buch und die drei in Paris befindlichen Chüan zur selben Ausgabe.

In diesem Teil habe ich seither besonders den Abschnitt über die Gestalt der Organe des ganzen Körpers übersetzt und mit der Literatur verglichen. Wie üblich sind Gewicht, Länge und - wo erforderlich - Umfang und Hohlmaße der einzelnen Organe angegeben. Längenmaße aus altchinesischen Werken, die im Sektionsmaterial aber wegen des fehlenden Tonus der Muskulatur immer etwas unsicher bleiben, benutzte Backiang Liang (1956) zu einem Vergleich mit heutigen Befunden. Ich führe hier als Beispiel die Gewichtsangaben an, die mir zuverlässiger bestimmbar erscheinen. Als Maßeinheiten dienen ein Chin, gewöhnlich übersetzt als chinesisches Pfund, und ein Liang, üblicherweise als Unze bezeichnet. Leider wissen wir nicht genau, welchen Gewichten in Gramm diese Einheiten zu verschiedenen Zeiten und in verschiedenen Gegenden Chinas entsprachen. Trotzdem kann man die Angaben auswerten, wenn man sie in Relationen ausdrückt. Ich habe z.B. alle Gewichtswerte der Organe in Liang umgerechnet und sie mit den uns bekannten Organgewichten in Gramm in Beziehung gesetzt. So erhalte ich für die Leber 68 Liang gegenüber $1500 \mathrm{Gramm}$, d.h. eine Proportion von 1 : 22. Für das Herz ist die Proportion $1: 25$, für die Lunge $1: 23$, für die Nieren $1: 18$, für den Darm $1: 17,6$. Ich will nicht behaupten, daß der Autor des Wan Ping Hui Ch'un solche Wägungen selbst ausführte, die entsprechenden Zahlen werden in der chinesischen Literatur seit alten Zeiten so angegeben. Es will mir aber scheinen, diese Proportionswerte liegen nahe genug beieinander, um daraus schließen zu können, daß sie auf reellen Befunden beruhen; indirekt beweisen sie, daß früher in China wirklich Sektionen vorgenommen worden sind. Die einzigen Ausnahmen von diesen 


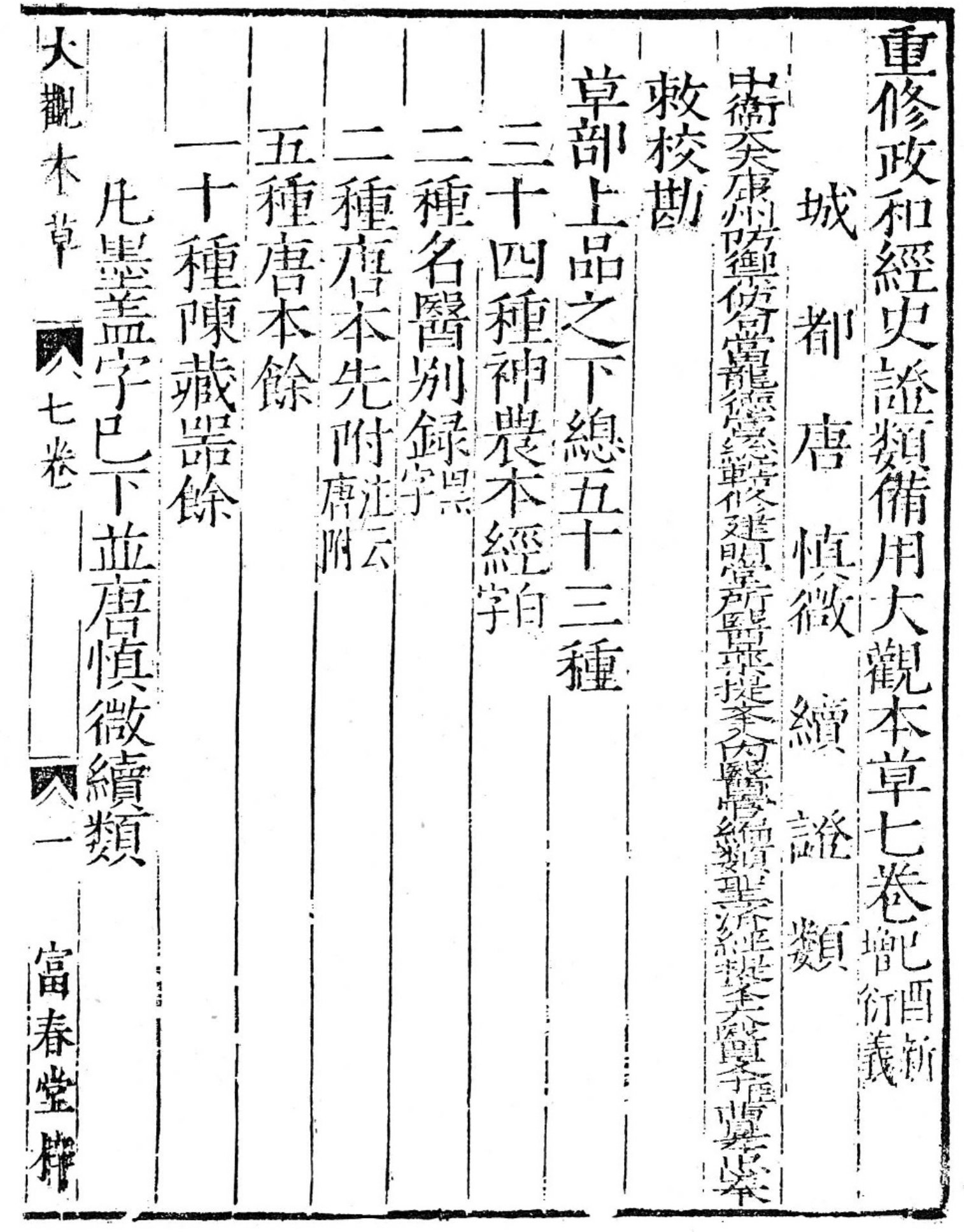

Abb. 2. Fol. 1r des siebenten Buches des Ta-kuan Pen-ts'ao von Tang Shen-weI 
recht ähnlichen Gewichtsrelationen betreffen den Magen, dessen Gewicht mit 46 Liang viel zu hoch angegeben wurde; entweder war er krankhaft verändert oder er wurde mit dem Inhalt gewogen, so daß sich eine Proportion von 1 : 3,2 ergibt. Die Milz soll mit 35 Liang fast halb so viel wie die Leber wiegen. Bei diesem Organ ist daran zu denken, daß einerseits die unterschiedliche Blutfülle, andererseits die Vergrößerung bei den weit verbreitet gewesenen Infektionskrankheiten einen zu hohen Gewichtswert verursachen konnte; die Proportion ist $1: 4,6$.

Den zweiten, Bilder und Beschreibungen von Arzneipflanzen enthaltenden Teil des Codex 350 habe ich seither bestimmen können als das siebente Chüan des Ta-kuan Pen-ts'ao von Tang Shen-wer. Der genannte Titel steht je auf der Faltzeile der Blätter. Ta-kuan ist die Regierungsperiode der Jahre 1107-1111. Dies ist also die Zeit der 1.Auflage. Der volle Titel läßt sich der 1.Zeile von fol.1r des Textes (Abb.2) entnehmen. Er lautet: Chung hsiu Cheng-ho Ching shih Cheng lei pei yung Ta-kuan Pen-ts'ao ch'i yüan. Die darin genannte Regierungsperiode Cheng-ho umfaßt die Jahre 1111 bis 1117. In dieser Zeit wurde auf kaiserlichen Befehl eine zweite revidierte Auflage des Werkes mit entsprechend angepaßtem Titel herausgebracht. Das Berner Exemplar ist ein nochmals etwas veränderter Nachdruck dieser Ausgabe. Leider läßt das Fehlen eines eigentlichen Titelblattes keine Datierung zu. Die Druckstöcke der insgesamt 49 Blätter sind 286 bis $290 \mathrm{~mm}$ breit und 195 bis $204 \mathrm{~mm}$ hoch. Der Text gliedert sich in die Faltzeile und zweimal 11 Schriftzeilen von je 21 Charakteren ohne Interpunktion; eingeschaltet sind 56 Abbildungen von Pflanzen, die teils einzeln, teils in Gruppen zu drei bis vier Stück angeordnet sind.

Vom Cheng lei Pen-ts'ao, d.h. «Nach Klassen geordnete Materia medica», gibt es viele Ausgaben. Eine reichhaltige Sammlung derselben besitzt die Kongreß-Bibliothek in Washington (D.C.) ${ }^{12}$. Der Vergleich dieser Nachdrucke und Bearbeitungen mit Photokopien des Berner Exemplares ergab jedoch keine Übereinstimmung. Indessen machte mich der Referenz-Bibliothekar der chinesischen Abteilung der genannten Bibliothek liebenswürdigerweise auf den Namen des Holzschneiders Fu CH'Un-T'ANG aufmerksam, der jeweils unten auf der Faltzeile aller Blätter des Berner Exemplares steht. Seine Lebenszeit ist zwar auch nicht genau zu datieren; ein der Kongreß-Bibliothek gehörendes Schauspiel, dessen Druckstöcke aus der gleichen Firma stammen, soll vermutungsweise in der Periode Wan-li (1573

12 Vgl. Report of the Librarian of Congress 1930 und früher. 
bis 1619) erschienen sein. Daraus ergäbe sich, daß die in Bern vorhandene Ausgabe des Ta-kuan Pen-ts'ao vielleicht gleichen Alters ist, wenigstens was das Erscheinungsdatum anbelangt. Der botanische und der anatomische Teil des Codex 350 könnten also um dieselbe Zeit nachgedruckt worden sein. Inhaltlich handelt es sich aber bei dem hier beschriebenen Pen-ts'ao um Wissen des 12. Jahrhunderts.

Der Inhaber des Lehrstuhles für Geschichte der Medizin in Peking, Professor LeE T'AO, gab 1954, einen kurzen Überblick über die Entstehungsgeschichte des Cheng lei Pen-ts'ao. Er hob besonders hervor, daß die Chengho-Ausgabe dieses Werkes nahezu fünfhundert Jahre im Gebrauch blieb. Man kann sogar sagen, daß sie während dieser Zeitspanne die bedeutendste Materia medica der Länder des Fernen Ostens war. Sie wurde erst nach 1597 allmählich durch das Pen-ts'ao kang mu verdrängt. $\mathrm{Daß}$ es sich bei dem Cheng lei Pen-ts'ao wirklich um ein historisch wertvolles Werk handelt, beweisen am besten die immer wieder erschienenen Nachdrucke. So sah ich in der Bibliothek des Bri ish Museum, London, eine Ausgabe aus dem Jahre 1920, betitelt Ch'ung hsiu Cheng-ho Ching shih Cheng lei Pen-ts'ao; ferner kam noch 1957 in Peking ein photolithographischer Nachdruck heraus, der nach einer Ausgabe von 1249 angefertigt ist; sein Titel lautet - fast gleich dem eben angeführten: Ch'ung hsiu Cheng-ho Ching shih Cheng lei pei yung Pen-ts'ao.

Zur näheren Charakterisierung des Werkes sei SARTON angeführt. Er schrieb (1931, S.247): Eine bedeutende Materia medica stamme vom Beginn des 12.Jahrhunderts, das Cheng lei Pen-ts'ao; es sei 1108 datiert und soll T'ANg SheN-weI, einen Arzt aus Shu in Ssu-chuan, zum Verfasser haben. Dieses Buch würde auch Ta-kuan Pen-ts'ao genannt, weil es während der Regierungszeit des Sung-Kaisers Hur Tsung vollendet sei, und zwar ein Jahr, nachdem dieser 1107 die Regierungsperiode Ta-kuan angetreten habe. Bedeutend sei das Werk, weil es Fragmente des Textes des legendären Buches Shen-Nung Pen-ts'ao ching enthalte, die in späteren gleichartigen Schriften als höchst wertvoll angesehen wären. Der Inhalt des Werkes sei gegliedert in die Kapitel: Kostbare Steine, Metalle, Kräuter, Getreide, Gemüse, Früchte, Bäume, Insekten, Fische, Vögel, Vierfüßer und Mensch. Nach Anführung einiger sorgfältig illustrierter Ausgaben aus den Jahren 1468, 1523, 1552 und 1654 hebt SARTon besonders hervor: «Es gibt auch eine Ming-Ausgabe, betitelt Ta-kuan Pen-ts'ao in 31 Chüan mit einem Vorwort aus der Sung-Zeit.» Dieser Hinweis scheint mir für das Berner Exemplar besonders wichtig, denn er könnte auch für dieses gelten. 
Sinn und Bedeutung der frühen chinesischen Werke über Heilmittel, die sowohl anorganische als auch pflanzliche und tierische Materialien umfassen, waren übrigens zum Teil gleich denen unserer alten Kräuterbücher, die sich ja auch nicht auf die Darstellung von Pflanzen allein beschränkten. Gerade so, wie deren botanischer Teil dazu dienen sollte, die nördlich der Alpen wachsenden Heilkräuter mit den mediterranen Pflanzen der antiken medizinischen Schriftsteller zu vergleichen, so sollten die Pen-ts'ao ermöglichen, Aussehen und Benennung der Arzneipflanzen aus verschiedenen Gegenden Chinas festzulegen.

Da den Fachleuten mit dem Neudruck von 1957 eine ziemlich alte Fassung des Cheng-ho Ching shih Cheng lei Pen-ts'ao leicht zugänglich ist, kann ich mich in der Analyse des Berner Teiles des Ta-kuan Pen-ts'ao kurz fassen, zumal ja nur eines der 30 oder 31 Chüan vorliegt, aus denen das ganze Werk bestehen soll. Alle 53 im siebenten Chüan angeführten Pflanzenarten sind älteren Werken entnommen. Für 34, also fast zwei Drittel, wird das klassische Kräuterbuch von Shen Nung als Quelle genannt. NeEdHam (1959, S.712) übersetzte dessen Titel Pharmacopoeia of the Heavenly Husbandman und setzte die Entstehung des Werkes in das 2. Jahrhundert unserer Zeitrechnung. Zwei Pflanzen stammen aus dem Ming I Pieh Lu (nach Needham: Informal Records of Famous Physicians, vielleicht aus der Periode der drei Königreiche oder aus der Chin-Dynastie, das würde heißen zwischen dem 3. und dem 5. Jahrhundert verfaßt). Zwei weitere Pflanzen sind dem T'ang-Pen-ts'ao Hsien fu und fünf dem T'ang Pen-ts'ao Yu entnommen; sie waren also schon in der Pharmakopöe der T'ang-Dynastie enthalten, was bedeutet, daß sie mindestens seit dem 7 . bis 9 . Jahrhundert als Heilmittel bekannt waren. Endlich sind noch zehn von CH'EN Ts'ANGCHI in seinem Werke Pen-ts'ao Shih I beschriebene Pflanzen in das siebente Buch des Ta-kuan Pen-ts'ao aufgenommen (Titel nach Needham, 1956, S.601: Omissions from Previous Pharmacopoeias, gleichfalls aus der T'angZeit, etwa 725 , verfaßt.)

Textlich bietet der Nachdruck von 1957 gegenüber dem Berner Exemplar nur sehr wenig Ergänzungen, dagegen sind darin alle Bildstöcke geändert. In der Wiedergabe der Blattstellung, der Blütenform und auch des Wurzelwerkes sind die Abbildungen des Berner Ta-kuan Pen-ts'ao eher steifer, also wohl ursprünglich einer früheren Ausgabe zugehörig. Übrigens sind sie doch nicht so schlecht, wie Fabricius Hildanus angab. Selbst bei bescheidenen botanischen Kenntnissen erkennt man leicht einzelne der Pflanzen an typischen Merkmalen, z.B. fol.19 die Typha latifolia, chinesisch P'u 


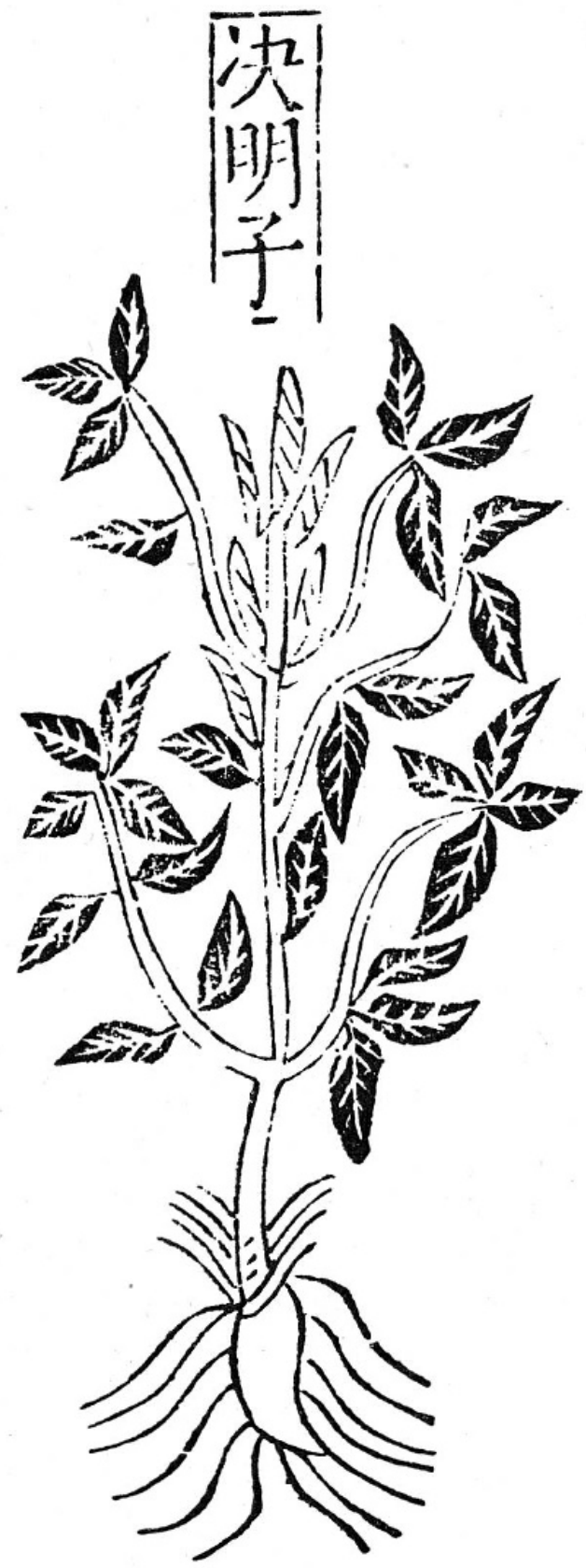

Abb. 3. Cassia obtusifolia aus dem Ta-kuan Pen-ts'ao von Tang Shen-weI 
huang. Interesse mag auch die Tatsache finden, daß einzelne der Pflanzenbilder dieser Ausgabe in spätere Werke übernommen wurden. НüвоттеR (1957), der seinem Buche Zeichnungen aus dem Pen-ts'ao kang mu beigab, bringt z.B. auf S.73 eine Orobanche ammophyla, chinesisch Jouts'ung-jung, die in Form und Anordnung ganz der im Berner Exemplar des Ta-kuan Pen-ts'ao (fol.17) gleicht. Dasselbe Bild dieser Pflanze ist auch in der oben zitierten Ausgabe des Cheng-ho Ching shih Cheng lei Pen-ts'ao von 1920 und in der von 1957 enthalten. Eine wesentliche Änderung scheint mir, daß in der Ausgabe von 1249 (Neudruck 1957) alle Figuren als Umrißzeichnungen schwarz auf weißem Grund mit schwarzen Blattrippen erscheinen, während im Ta-kuan Pen-ts'ao eine Reihe von Bildern in umgekehrter Manier dargestellt ist, also mit schwarzen Blättern und weiß ausgesparten Rippen. Die in Abb.3 wiedergegebene Zeichnung der Cassia obtusifolia s. Tora, chinesisch Chüeh-ming-tze, mag als ein Beispiel dafür dienen, daß auf diese Weise ein recht ansprechender Effekt erzielt werden kann.

Ganz ähnlich bewertete Haller (1771, Vol.I, S.5) solche Bilder in dem Bericht über ein in seinem Besitz befindliches botanisches Werk aus China; Titel und Verfasser kannte er allerdings ebensowenig wie die Datierung. Es soll 105 Abbildungen enthalten haben, die zwar roh, aber doch immerhin noch besser seien als die von Dodonaeus herausgegebenen, nach denen niemand die Pflanzen erkennen könne. Nach den chinesischen Abbildungen dagegen sei es ihm möglich gewesen, viele Pflanzen zu bestimmen; dann heißt es weiter: Die Bilder zeugen von einer gewissen Kunstfertigkeit; es gibt nämlich solche, in denen der Holzschneider in den schwarzen Blättern weiße Rippen genau und nicht ohne Schwierigkeiten dargestellt habe also gerade die vorstehend vom Ta-kuan Pen-ts'ao beschriebene Art. Des Vergleiches wegen wäre es interessant, festzustellen, welches chinesische Buch über Pflanzenkunde zu Hallers Bibliothek gehörte. Meine diesbezüglichen Bemühungen blieben bisher erfolglos. Die Spuren führen nach Paris, doch müßte man schon alle eventuell in Frage kommenden Werke am Standort einsehen können, um etwa durch das Exlibris oder gar durch eine handschriftliche Notiz Hallers die frühere Zugehörigkeit zu dessen Büchersammlung zu sichern.

Etwas genauer sei in diesem Zusammenhang noch über HaLlers Kenntnis der chinesischen Literatur berichtet. Tatsächlich findet man in allen vier Bibliothecae jeweils im Anschluß an den Abschnitt «Arabes» chinesisches Schrifttum angeführt, meistens zitiert nach Katalogen der großen 
ausländischen Büchersammlungen wie der des Königs von Frankreich, des Britischen Museums, der Bodleyana oder aus dem noch immer lesenswerten Werk des Paters Du Halde über China aus dem Jahre 1735. Auf eine weitere wichtige Quelle von HaLLERs bibliographischen Kenntnissen chinesischer Werke wurde ich besonders aus dem Nachtrag am Ende des zweiten Bandes der Bibliotheca anatomica aufmerksam. Sie betrifft einen Mann, der wohl verdiente, auch in Ärztekreisen nicht vergessen zu werden: CHRIStopH Gottrleb von Murr aus Nürnberg. Sein Name ist in den medizin-historischen Lexika nicht zu finden, denn voN MURR war weder Arzt noch Naturwissenschafter, sondern Jurist. Wer seine Adnotationes ad Bibliothecas Hallerianas zur Hand nimmt, wird darin gleich zu Anfang bei den Nachträgen zur Bibliotheca botanica auf chinesische Schriftzeichen stoßen; später, am Beginn der Ergänzungen zur Bibliotheca anatomica, finden sich wieder chinesische Charaktere. Merkwürdigerweise ist dort der Titel des Pen-ts'ao kang $m u$ angeführt, allerdings in einer von der heutigen recht abweichenden Transkription. Eigentlich handelt es sich bei diesem Werk ja um ein Kräuterbuch, aber da die chinesische Materia medica auch tierisches Material zum Gegenstand hat, so kommen im Hinblick auf die vergleichende Anatomie ganze Tabellen chinesischer Namen von Vierfüßern auf S.27 zum Abdruck. Dieselbe Tafel findet sich schon in dem durch von MurR herausgegebenen Journal zur Kunstgeschichte und zur allgemeinen Literatur (4. Teil, 1777, S.163). Nach den im 6. Teil der gleichen Zeitschrift veröffentlichten Briefen Hallers an von MurR sandte dieser ihm chinesische Holzschnitte, offenbar von Pflanzenbildern, denn HALLER bedankte sich dafür am 22. April 1775 und schrieb dazu: «Ich besitze ein Kräuterbuch in dieser Sprache, ungefähr von eben der Art.» Im Frühjahr 1777 diskutierten HaLLER und voN MURR in ihren Briefen die Entstehungszeit bestimmter medizinischer Werke. Murr hielt an den alten Datierungen der chinesischen Chronologie fest, während HALLen der Ansicht war, daß die Zeitrechnung der Chinesen «zu hoch hinauf steigt und von ihren heutigen Schriftstellern selbst verlassen wird» (Brief HALLERs vom 2. März 1777).

Was war das für ein seltsamer Mann, der sich im 18. Jahrhundert so eingehend mit chinesischer Literatur befaßte? Ich zitiere nach der Allgemeinen Deutschen Biographie, Band 23 vom Jahre 1886: Christoph Gottuieb vón MurR wurde am 6.August 1733 in Nürnberg geboren und starb dort am 8. April 1811 als pensionierter Zoll- und Wagamtmann. Er erhielt im Nürnberger Gymnasium eine recht gründliche Kenntnis der alten Sprachen und wurde zusätzlich in Geographie, im Hebräischen und Französischen unter- 
richtet. Studiert hat er 1751 bis 1754 ausschließlich in Altdorf und zwar als Jurist, wo ihn der Staatsrechtslehrer Heumann besonders anregte. Dessen Bibliothek ermöglichte ihm auch, sein Wissen auf die Philosophie, Mathematik, Naturwissenschaften, Archäologie und Geschichte auszudehnen. Noch vor seiner Promotion, im Jahre 1753, begann er die Vorarbeiten zu umfassenden literarischen Werken, so einer Bibliotheca glottica universalis, einer Bibliotheca mathematica universalis, einer Bibliotheca ophthalmographica, später auch einer Bibliotheca rhetorica und einer Bibliotheca dimicatorica, also wohl der Befestigungskunst und Strategie, für die voN MurR in jungen Jahren großes Interesse hatte. In Straßburg, wohin ihn 1756 weitere Studien führten, kam er in Berührung mit Jesuiten, für die er Zeit seines Lebens große Sympathien bewahrte, obwohl er selbst Protestant war und blieb. Weitere Stationen seiner Studienreise waren Rotterdam, Amsterdam, Leiden, Utrecht, London, Oxford und Cambridge. Überall nahm er Beziehungen zu den bedeutendsten Gelehrten, Künstlern und Staatsmännern auf; aus jenen Jahren stammen die Vorarbeiten zu einer 1770 erschienenen Bibliothèque de peinture, de sculpture et de gravure. Nach einer kurzen Zwischenzeit in Nürnberg ging er $1758 \mathrm{zu}$ einem fast einjährigen Aufenthalt nach Wien, von wo er über Oberitalien heimkehrte. 1761 weilte er ein zweites Mal elf Monate lang in England, auf der Rückreise besuchte er Hamburg. Dann trat er endgültig in seiner Heimatstadt das Amt an, in das er schon 1760 gewählt war, er wurde Zoll- und Wagamtmann.

Die überaus reiche literarische Tätigkeit voN MurRs betraf verschiedenste Wissensgebiete. Vornehmlich befaßte er sich mit Geschichte, Archäologie und Kunstgeschichte, aber auch zur Geschichte der Mathematik, der Medizin und der Naturwissenschaften hat er Beiträge geliefert, ferner hatte er als Sprachgelehrter einen angesehenen Namen. Dem Zeitgeiste folgend gab er wissenschaftliche und unterhaltende Journale heraus, in denen sich die Niederschläge seiner weitverzweigten Verbindungen mit Gelehrten und zu den als Missionaren in fernen Landen tätigen Jesuiten finden. Bemerkenswert für das hier behandelte Thema ist sein Vorschlag, die chinesischen Schriftzeichen für eine Universalsprache zu verwenden, wozu sie durch ihre genauen Definitionen bestens geeignet wären. Schließlich sei erwähnt, daß voN MurR später Streit mit Lessing hatte wegen dessen Schrift über Laokoon; er erwies sich dabei als selbstbewußt und überheblich, machte also nicht gerade gute Figur.

Chr.G.von Murr trat mit Haller erst von 1771 an, also in dessen letzten Lebensjahren, in Briefwechsel, hauptsächlich wegen der Bibliothecae, 
zu denen er Ergänzungen und Nachträge lieferte. HALLER publizierte selbst noch Teile davon. Am ausführlichsten sind die Briefe hinsichtlich der in europäischen Büchersammlungen vorhạdenen chinesischen Werke; ihre Zahl war schon damals recht erheblich. Unter anderem teilte von MurR ein ausführliches Verzeichnis mit Inhaltsangaben solcher Bücher aus dem Britischen Museum mit, ferner berichtete er über die Bestände der Königlichen Bibliothek in Paris an Hand von Fourmonts Grammatica Sinica. In diesen Briefen finden sich häufig chinesische Schriftzeichen, die aus Büchern ausgeschnitten und in die Briefe eingeklebt sind. Da die Transkription in Hallers Bibliothecae auf heute ungewöhnliche Weise vorgenommen wurde, ist es nicht immer leicht zu erkennen, um welche Werke es sich handelt. Nur die chinesischen Charaktere der Originalbriefe und Kataloge wie der von Fourmont und Courant geben die Möglichkeit zu genauen Bestimmungen.

Ich bin sicher, in einer der vielen HALLER-Biographien gelesen zu haben, daß dieser selbst aus China Briefe erhalten hätte, doch kann ich mich des betreffenden Autors nicht mehr erinnern. Der Katalog der Haller-Briefe gab mir keine derartigen Hinweise. So wäre wohl möglich, daß die chinesischen Schriftzeichen in den Briefen von MurRs eine derartige Angabe veranlaßten. Wie sehr sich HALLER in die chinesische Welt einzuleben versuchte, läßt am besten sein Altersroman Usong erkennen. $\mathrm{Zu}$ zeigen, daß HALLER nicht nur durch literarische Interessen, sondern auch aus fachwissenschaftlichen Gründen am chinesischen Schrifttum ernsthaft Anteil nahm, war eines der Ziele bei der Zusammenstellung vorstehender Angaben aus seinen Werken und seinem Briefwechsel. Am wichtigsten aber war mir, über zwei medizinische Werke aus China zu berichten, weil sie zu den ersten derartigen Büchern gehören müssen, die nach Europa kamen. 


\section{Schriftum}

Manuskripte:

Guilelmi Fabricir Hildani epistolae de rebus medicis, Burgerbibliothek Bern, Cod. 495-4.97.

Haller A. De scriptis ineditis Guil. Fabricii Hildani, fol., Burgerbibliothek Bern, Mss. Haller 21.

Hontinus S. Handschriftlicher Katalog der Bibliotheca Bongarsiana, Burgerbibliothek Bern.

Druckwerke:

Backiang Liang, Contributions of the Ancient Classical Chinese Medicine to Anatomy and Physiology, J. Pakistan Med. Assoc. 6 (1956).

Becker G. Wilhelm Fabricius von Hilden, 2. Auflage, in H. Strangmeier, Wilhelm Fabry von Hilden, Wuppertal-Elberfeld 1957.

Bцösch H. Die Stadt- und Hochschulbibliothek Bern, Bern 1932.

Courant M. Catalogue des livres chinois, coréens, japonais etc., Tome 2, Paris 1910.

Du Halde J. B. Description de l'Empire de la Chine, vol. 3, Paris 1735.

FABRY W. Von der Fürtrefflichkeit und Nutz der Anatomy, 2. Auflage, Aarau/Leipzig 1936.

Fourmont St. Linguae Sinarum grammatica ... item Sinicorum Regiae Bibliothecae librorum catalogus, Lutetiae Parisiorum 1742.

Hagen H. Catalogus codicum Bernensium, Bernae 1875.

Haller A. v. Bibliotheca botanica, Vol. I, Tiguri 1771.

- Bibliotheca anatomica, Vol. I, Tiguri 1774.

- (anonym) De scriptis ineditis Guilelmi Fabricii Hildanae prope Coloniam nati: quem vulgo Hildanum vocant. Relationes de libris novis. Fasc. XII. Gottingae 1754.

Hintzsche E. Über anatomische Tradition in der chinesischen Medizin, Mitt. Naturf. Ges. Bern, N.F. 14. (1957).

Huard P. et Wong, Bio-Bibliographie de la médecine chinoise, Bull. Soc. Etud. Indochin., N. S. 31 (1956).

Huard P. et M. Wong, La médecine chinoise au cours des siècles, Paris 1959.

Hёвоттев F. Chinesisch-tibetanische Pharmakologie und Rezeptur, Ulm 1957.

LeE T'Ao, Achievements of Chinese Medicine in the Northern Sung Dynasty (960-1127 a.D.), Chin. Med. J. 72 (1954).

Mathews R. H. A Chinese-English Dictionary, Shangai 1931.

Murr Cн. G. v. Adnotationes ad Bibliothecas Hallerianas, Erlangen 1805.

- Journal zur Kunstgeschichte und zur allgemeinen Literatur, 4. und 6. Teil, 1777-78.

Needham J. Science and Civilisation in China, Cambridge, vol. 2 (1956), vol. 3 (1959).

SARTON G. Introduction to the History of Science, vol. 2, Washington 1931.

Sinner J. R. Catalogus Codicum Mss. Bibliothecae Bernensis, 3 Vol., Bernae 1760-72.

Wallnöfer H. und A.v. Rottauscher, Der goldene Schatz der chinesischen Medizin, Stuttgart 1959.

Wong K. CH. and L. T. Wu, History of Chinese Medicine, Shanghai 1. Auflage 1932, 2. Auflage 1936.

Report of the Librarian of Congress 1916, 1920, 1925, 1927, 1930. 